der Cyklohexylhalogenide mit Cyanessigester und mit Malonester; $\mathrm{B}$ r u $\mathbf{n}$ e ${ }^{55}$ ), Cyklohexanol- und -hexandiolderivate aus Cyklohexen; $\mathrm{H}$ a $\mathrm{ll}$ e $\mathbf{r}$ und $\mathrm{M}$ a $\mathrm{r} \mathrm{ch}^{56}$ ), Einwirkung von aromatischen Aldehyden auf Hexanolnatriumverbindungen und von Alkoholaten auf Hexanone.

Die zu diesen zahlreichen Synthesen verwendeten cyklischen Alkohole und Ketone bzw. deren Homologe, von denen nur 1,3-Methylcyklohexanon vor Jahren von $\mathrm{W}$ a $11 \mathrm{a} \mathrm{ch}$ durch Spaltung des Pulegons dargestellt war, sind erst in den letzten Jahren durch die von $\mathrm{S}$ a b a t i e r und seinen Mitarbeitern angegebene Reduktionsmethode zugänglich geworden. Ihr Verfahren besteht bekanntlich darin, daß Dämpfe von Phenolen, wie Phenol selbst, den Kresolen usw., mit H-Gas gemischt über auf $160-220^{\circ}$ erhitztes Nickelpulver geleitet werden, je nach der Reaktionsfähigkeit des betreffenden Phenols. Außer den Hexahydrophenolen (Cyklohexanolen) entstehen in geringer Menge auch die zugehörigen Ketone. Holleman, van der L a a $n$ und S l i j p e $\mathrm{r}^{57}$ ) untersuchten die Hydrierungsbedingungen des Phenols näher und erhielten bei $140-150^{\circ}$ ein Gemenge von Alkobol und Keton, aus dem sie letzteres durch Kondensation mit Benzaldehyd entfernten; bei dreimaligem Uberleiten über $\mathrm{Cu}$ mit Luft bei $300^{\circ}$ erhielten sie Cyklohexanon, bei $330^{\circ}$ entstand viel Cyklohexan. Neuerdings ist diese Reaktion auf einige höhere Phenole ausgedehnt worden; die dabei erhaltenen Resultate sind insofern von Interesse, als manche der dargestellten cyklischen Alkohole in Beziehungen zu gewissen Torpenalkoholen zu stehen scheinen. So erhielt B r u ne ${ }^{58}$ ) aus Thymol einen mit Menthol stellungsisomeren Alkohol Thymomenthol, der sich durch das Succinat oder Phthalat hindurch in ein isomeres $\beta$-Thymomenthol überführen ließ. Beide Isomeren lieferten bei der mit Chromsäure vorgenommenen Oxydation dasselbe Keton, Thymomenthon, das jedoch reduziert nur $\beta$-Thymomenthol zurückgab.

Aus Carvacrol entstanden nach derselben Methode ebenfalls zwei isomere Carvacromenthole $\mathrm{H}$ a $\mathrm{Il}$ e $\mathrm{r}^{59}$ ) und $\mathrm{M}$ a $\mathrm{r} \mathrm{t}$ i $\mathrm{n} \mathrm{e}^{60}$ ) erhielten aus dem in der Seitenkette ungesättigten Pulegon durch schrittweise Reduktion erst das gesättigte Keton Pulegomenthon, weiter den Alkohol Pulegomenthol; dieser erwies sich als ein Gemenge von gewöhnlichem 1-Menthol mit festem $a$ - und flüssigem $\beta$ Pulegomenthol. Die Autoren geben genau die Versuchsbedingungen, wie Temperatur, Dauer der Reaktion, Schnelligkeit des zufließenden Pulegons usw. an. Nach Ma $i l$ he $\mathrm{e}^{61}$ ) lassen sich nach diesem Verfahren auch Oxime zu Aminen reduzieren.

Zusammenfassend behandelten die Entdecker

55) Ann. Chim. 6, 200, 288 (1905).

56) Compt. r. d. Acad. d. sciences 140, 127, 130 , 474 (1905).

57) Recueil trav. chim. Pays-Bas 24, 19 (1905). (1905).

${ }^{58}$ ) Compt. r. d. Acad. d. sciences 140, 252, 792 (1905).

9) Compt. r. d. Acad. d. sciences 14, 1245

$\left.{ }^{60}\right)$ Compt. r. d. Acad. d. sciences 140, 1298 (1905).

61) Bll. Soc. chim. Paris (3) 33, 962 (1905).
Sabatier und Senderens62) ihre Methode in einem vor der Pariser Chemischen Gesellschaft gehaltenen Vortrage.

(Schiuf folgt.)

\section{Über die Vorgänge im Gloverturm und in den Bleikammern.}

\author{
Von G. LUNGe. \\ (Eingeg. d. 18.10. 1906.)
}

Es ist gewiß zu begrüßen, wenn an der in den letzten Jahren geführten Erörterung über den Bleikammerprozeß sich verschiedene Stimmen beteiligen, sofern diese in einer oder der anderen Richtung auf Sachkenntnis Anspruch erheben können. Man muß aber dann in erster Linie verlangen, daß die Teilnehmer an der öffentlichen Diskussion die Arbeiten der anderen Teilnehmer gründlich studieren und wirklich zu verstehen suchen. Wenn sie dieser Forderung nicht entsprechen und vielmehr die Ansichten derer, die sie bekämpfen wollen, mißverstehen und entstellen, so verursachen sie nicht nur ihren Gegnern durch die Notwendigkeit einer Berichtigung eine unnötige Belästigung, sondern auch dem gesamten Lesepublikum einen Verlust an Zeit und nehmen in den Zeitschriften den immer knapper werdenden Raum zu Unrecht in Anspruch.

Diesen Vorwurf wird man dem Vortrage nicht ersparen können, den Herr Dr. M. N e u m a $\mathrm{n} \mathbf{n}$ zu Cronberg i. T. bei der letzten Hauptversammlung des Vereins deutscher Chemiker gehalten hat und der in dieser $Z .19,1702 \mathrm{ff}$ (1906) abgedruckt ist. Ich will mich nicht darauf einlassen, die Verbesserungsvorschläge dieses Herrn für den Gloverturm zu kritisieren, von denen er selbst zugibt, daß sie den meisten Fachgenossen paradox erscheinen werden. Die in meinen Jahren noch verfügbare Arbeitskraft muß ich für andere Aufgaben reservieren, die ich für dankbarer halte. Aber leider zwingt mich der Grundsatz: qui tacet, consentire videtur, zur Abwehr der zahlreichen, natürlich unbeabsichtigten, aber nach meiner Ansicht durchaus nicht entschuldbaren Entstellungen meiner Ansichten, die sich in M. N e u m a n n s Aufsatz vorfinden.

Gleich auf der ersten Seite (S. 1702) findet sich eine ganze Anzahl solcher Entstellungen. Es ist schon auffallend, daß $\mathbf{N}$ e u $\mathrm{m}$ a $\mathbf{n}$ bei der Erwähnung der bei Kammertemperaturen vollständigen Dissoziation des $\mathrm{N}_{2} \mathrm{O}_{3}$ nur von, ,Ram s a y und anderen spricht", welche diese aufgefunden und $\mathrm{m}$ i c $\mathrm{h}$ zur Anderung meiner früheren Kammertheorie veranlaßt haben sollen, wobei es also nicht herausspringt, daß jene Dissoziation erst durch die von $\mathrm{m}$ i r mit $\mathrm{P}$ or s c h $\mathrm{n}$ e w angestellten exakten Arbeiten endgiiltig nachgewiesen worden und erst infolge dieser Arbeiten bis auf die neueste Zeit ( $\mathrm{R} \mathrm{a} \mathrm{s} \mathrm{c} \mathrm{h} \mathrm{i} \mathrm{g)} \mathrm{ohne} \mathrm{Widerspruch} \mathrm{angenommen} \mathrm{wor-}$ den ist. Aber geradezu falsch ist es, wenn es unmittelbar darauf heißt, ich habe durch eine,,fast ganz neue, mit B e r l aufgestellte Theorie (dicse

62) Bll. Soc. chim. Paris (3) 33, Beiheft I (1905). 
Z. 19, 883 [1906]) überrascht, die auf der alleinigen Wirksamkeit des bisher mit aller Macht verfehmten Peroxydes gegründet ist". Das ist nicht nur falsch, sondern einfach widersinnig, denn das angeblich bis dahin von mir verfehmte Peroxyd kommt in der von mir seit 1894 und in meinem "Handbuche" von 1903 durchgeführten Theorie überall als absolut notwendig neben NO vor und ebenso kommt es auch in meinen jetzigen Anschauungen zur vollen Geltung.

Nur so viel ist richtig, daß ich an einigen Stellen des Handbuches, wo von Stickstoffperoxyd die Rede ist, nur dasjenige meine, welches über das Verhältnis $\mathrm{NO}_{2}+\mathrm{NO}$ hinausgeht und welches ich z. B. auf S. 672 des "Handbuches" als ,freies“" Peroxyd bezeichne. Der denkende Leser wird diese Einschränkung auch da, wo sie nicht ausdrücklich gemacht ist, selbst machen.

Der von $\mathrm{N}$ e u m an $\mathrm{n}$ aus dem Zusammenhange gerissene Satz auf S. 670 meines ,Handbuches" bezieht sich auf ältere Anschauungen, was doch wahrlich deutlich genug daraus erhellt, $\mathrm{daB}$ das NO an einer Menge von Stellen meines Handbuches als wesentlich eingeführt wird, wovon ich nur S. $672,673,674,675$ anführen will. Wie kann M. $\mathrm{N}$ e u $\mathrm{m}$ a $\mathrm{n} \mathrm{n}$ weiterhin auf $\mathrm{S} .1702$ von einer ,fast radikalen Meinungsänderung $L$ u $n g$ e s" reden und es als seinen Befund herstellen, daß ,es möglich sein sollte, die Zwischenstufe der Sulfonitronsäure in die alte Theorie einzureihen", nachdem i c h mit B e r l in Nr. 15 der Zusammenfassung unserer Ergebnisse (S. 894) es mit dürren Worten ausgesprochen hatte, $\mathrm{da} B$ die Einschiebung dieser von uns als solche $\mathrm{n}$ a chg c w i esenen Zwischenstufe die alleinige wesentliche Änderung in meiner alten Theorie bedeute! Hier liegt denn doch, ich will nicht sagen eine absichtliche Entstellung, aber ein wirklich unverzeihliches Mißverständnis von seiten $\mathrm{M}$. $\mathrm{N}$ e $\mathrm{um}$ an $\mathrm{ns}$ vor.

Wenn also Neumann, immer noch auf S. 1702, meint, er wolle mir treuer bleiben als ich selbst, weil meine alte Theorie allen Anforderungen gerecht werde, so ist er päpstlicher als der Papst, womit ich natürlich nicht gesagt haben will, daß ich für meine Theorien den Anspruch der Unfehlbarkeit erhebe. Wie wenig ich mich vielmehr davor scheue, frühere Irrtümer ganz offen einzugestehen, habe ich in dieser Z. 18, 62 (1905) gezeigt, auf die Gefahr hin. daB mir spätere Gegner, die wohl ebenso wenig unfehlbar sind als ich, daraus einen Strick drehen möchten.

Noch eine weitere auf S. 1702 zu findende Darstellung $N$ e $u \operatorname{mann}$ s ist ganz unrichtig, als ob nämlich L e B 1 a n c durch seine Mitteilungen über das Verhalten des $\mathrm{N}_{2} \mathrm{O}_{3}$ gegen Alkalien einem meiner die (angeblich) neue Theorie begründenden "Axiome" den Boden entzogen habe. Abgesehen davon, daB B e r l und $\mathrm{i} h \mathrm{~h}$ nicht den sonderbaren Lapsus begangen habcn, eine tatsächliche Beobachtung (diese Z. 18, 858 [1905]) als "Axiom" zu bezeichnen und abgesehen davon, daß gerade in diesem Falle unsere Beobachtungen mit denen von $\mathrm{R}$ a s ch ig in dieser $\mathrm{Z}$. 18, 1291 (1905) übereinstimmen (wir fanden beide, daß das Gemenge von $\mathrm{NO}+\mathrm{NO}_{2}$, welches $\mathrm{R}$ as chig für $\mathrm{N}_{2} \mathrm{O}_{3}$ hält, von Natronlauge nicht unverändert aufgenommen worde, daß vielmehr ein Teil des NO nicht zur Ab- sorption kommt und daher in der Natronlauge $\mathrm{NO}_{2}$ als im Überschuß aufgenommen erschieint), können die Beobachtungen $\mathrm{L}$ e $\mathrm{Bl}$ a n es durchaus nicht als im Widerspruche mit $L$ u $\mathrm{n}$ e und $B$ e r 1 und mit $\mathbf{R}$ a s c hi g stehend hingestellt werden. Hier hat sich $M$. N e $u m$ an vielleicht durch kurze und ungenaue Auszüge aus L e B l a n c s Vortrag vor der Bunsengesellschaft täuschen lassen. Aus der ausführlichen Wiedergabe desselben in der $Z$. f. Elektrochem. 12, $541 \mathrm{ff}$. (1906) kann man entnehmen, daß L e B l a n o nicht nur in keinem Punkte den Ergebnissen von $\mathrm{B}$ er $\mathrm{l}$ und $\mathrm{m}$ ir widerspricht, sondern diesen ausdrücklich zustimmt. Es heißt auch bei ihm $\mathrm{S}$. 543, daß man bei Verwendung von Lauge als Absorptionsmittel für NO. haltige Gase leicht einen ÜberschuB von Nitrat gegenüber dem Nitrit erhält. Darïber, daß $\mathrm{N}_{2} \mathrm{O}_{3}$ nur ,in kleinen Mengen eine intermediäre Existenz führt", war bei der Diskussion über jenen Vortrag alles einig, und das ist ja 1894 von $\mathrm{m} \mathrm{i} \mathrm{r}$ und Porschnew, sowie neuerdings ron $\mathrm{Dix}$, und $P$ eterkin, quantitativ verfolgt worden. Diese Mengen sind bei gewöhnlicher Temperatur sehr gering, und über $50^{\circ}$, also bei Bleikammertem. peraturen, unmerklich klein. Ganz selbstverständlich schließt das nicht aus, daß bei dem Freiwerden von $\mathrm{N}_{2} \mathrm{O}_{3}$, z. B. ans Nitrosylschwefelsäure, dasselbe e in e n A u g e n b 1 i c k existiert, und daß gerade diese minimale, vorübergehende Bildung von $\mathrm{N}_{2} \mathrm{O}_{3}$ die Reaktionen des stöchiometrischen Gemenges $\mathrm{NO}+\mathrm{NO}_{2}$ am besten erklärt; das steht genau mit diesen Worten gerade in meiner früheren Abhandlung, diese Z. 18, 68 (1905). Dort wird aber eben auf den fundamentalen Unterschied dieser Anschauung von der von $R$ a $\& \mathrm{c} \mathrm{h}$ i g hingewiesen, der sich nicht mit solchen minimalen, gar nicht direkt nachweisbaren Mengen von $\mathrm{N}_{2} \mathrm{O}_{3}$ begnügt, sondern geradezu behauptet, daß in der Bleikammer die Gleichung : $2 \mathrm{NO}+\mathrm{O}=\mathrm{N}_{2} \mathrm{O}_{3}$ die einzig richtige und jede andere falsch sei, was ihn dann sogar zur Leugnung des Gesetzes von A vogadro führte.

Wir kommen nun zum Gloverturm. Da soll ich nach M. N e u m ann (S. 1703) Herm R a s c h i g unrecht getan haben, indem ioh dessen (in dieser Z. 18, 1315 [1905]) sehr emphatisch ausgesprochene und offensichtlich von ihm (und ausgesprochenermaßen von $\mathrm{Neu}$ a $\mathrm{n} n$ ) als ganz $\mathrm{n}$ e u angesehene Anschauung, wonach der Gloverprozeß ,genau derselbe wie der Bleikammerproze $B^{\prime c}$ sei, als etwas Selbstverständliches und als Gemeingut der Fachwelt bezeichne. Zwar $\mathrm{mu} ß \mathrm{~N}$ e u $\mathrm{m}$ a $\mathbf{n} \mathbf{n}$ selbst zugeben, daß diese Anschauung schon in meinem Handbuche ausgesprochen sei, aber die seines Wissens ,einzigsten" (dieser schöne Superlativ rührt nicht von mir her) Stellen bezögen sich ,zweifelsohne" nur auf die Glovergleichung IIb. Das ist von vornherein eine ganz willkührliche Annahme; aber man braucht in meinem Buche gar nicht weit zu suchen, um noch andere Belegstellon dafür «u finden, daß $R$ as chigs Anschauung nicht neu war. $\$ .674$ heißt es : ,In der Tat sind in diesem Teile der Kammer die Bedingungen noch ganz analog den in dem unmittelbar vorhergehenden Gloverturme herrschenden", und S. 675: „Dies ist natürlich genau dieselbe Reaktion, wie sie in dem unmittelbar vorhergehendem Raune, d. h. im Gloverturme, vor sich geht. Die Bedingungen sind in beiden Fällen auch 
vollkommen dieselben nsw." Wenn ich an der ersten angeführten Stelle hinzufüge, daß im hin t e r e $n$ Teile des Kammersystems, wo die Temperatur niedriger und viel weniger $\mathrm{SO}_{2}$ vorhanden ist, die Bedingungen andere sind, so wird das doch niemand bestreiten können, der eine Ahnung von den Verhältnissen hat, und auch $\mathrm{N}$ e u m a n n tut das nicht (s. unten). $\mathrm{Zu}$ übersehen ist doch auch nicht, daß ich auf S. 594 die Folgerung besonders ablehnen zu müssen glaube, daß man auf Grund der an jener Stelle von mir entwickelten Anschauungen statt der Kammern nur einen hinreichend großen Gloverturm anzuwenden brauche und dafür meine Gründe anführe. Darin liegt es doch gewiß mit aller Deulichkeit, daß die Vorgänge im Glover und in der Kammer von gleicher Art seien und keinerlei sprungweise Änderung beim Übergange aus dem Glover in die Kammer stattfindet. Ich habe mir nicht die, gewiß unnötige, Mühe genommen, noch weitere Belege dafür in meinem dicken Buche aufzusuchen; das Gesagte ist wahrlich genügend.

Von dem vielen Unbegreiflichen, das in $\mathrm{N}$ e $\mathrm{u}$ m a n n s Vortrag steht, ist es vielleicht das Unbegreiflichste, daß er, der auf S. 1703 als ,einzigste“" solcher Belege die Stellen S. 197 und 594 des ,Handbuches" angeführt hat, auf S. 1704 ebenfalls S. 675 dieses Buches zitiert und dazu folgende Bemerkung macht : „Er (d. h. L. u n g e) würde sonst nicht behaupten können, die Bedingungen im ersten Teile des Kammersystems und imGloverturm seien nicht nur vollkommen dieselben" usw. Es ist wirklich nicht leicht, sich stärker ins Gesicht zu schlagen, was die angebliche Ungerechtigkeit meiner Reklamation gegenüber $R$ a s c h i g betrifft.

An allen diesen Stellen meines Buches ist also ausdrücklich die Identität der Bedingungen und mithin auch der Vorgänge mit denen des Gloverturmes nur für den v o r d e r e n Teil des Kammersystems ausgesprochen, in dem ja allerdings die meiste Säure erzeugt wird. Daß diese Identität für die $h$ in te ren Kammern ebenfalls bestehe, sagt $R$ as ch i g nicht, aber auch nicht das Gegenteil, und man könnte vielleicht die Annahme einer Identität zwischen den Vorgängen im Glover und denen in a 11 e $\mathbf{n}$ Teilen des Kammersystems aus seinem Aufsatze in dieser Z. 18, 1316, erste Spalte oben (1905) folgern. Nach meinem Gefühle meint aber auch $R$ a s c hig nicht, daß die Vorgänge bei den doch sicher in bezug auf Temperatur und auf $\mathrm{Zu}$ sammensetzung der Gase durchaus abweichenden Verhältnissen in der e $\mathrm{r}$ s te $\mathrm{n}$ und der let $\mathrm{z}$ te $\mathrm{n}$ Kammer identische seien.

M. Ne u m a n n widerspricht direkt der Annahme einer solchen Identität für das ganze System (Seite 1706, zweite Spalte oben), und hierin stimmen wir nun ganz überein, wie die soeben von mir aus meinem Buche von 1903 gegebenen Zitate erweisen, auf die ich mich auch in meinen seitherigen Ver. öffentlichungen durchaus gestützt habe. Lim so weniger Berechtigung hat $\mathrm{Neu} \mathbf{m}$ a $\mathbf{n}$, mir $\mathrm{zu}$ insinuieren, daß ich der Anschauung einer Identität der Vorgänge i m g a $\mathrm{n}$ z e $\mathrm{n} S \mathrm{y}$ s t e m e (wenn sie wirklich die von $R$ as chi $g$ ist), neuerdings beigetreten' sei.

Hiermit glaube ich alle Stellen besprochen $\mathrm{zu}$ haben, an denen Herr M. $\mathrm{N}$ e u $\mathrm{m}$ a $\mathbf{n} \mathrm{n}$ mir unter ganz mißverständlicher Darstellung meiner Ansichten Irrtümer nachzuweisen gesucht hat, und ich glaube, in allen Fällen die mir gemachten Vorwürfe bis auf den letzten Punkt widerlegt zu haben. Auf eine Besprechung der von ihm aufgestellten Vorschläge für Verbesserungen im Kammerverfahren gehe ich, wie schon im Eingange bemerkt, nicht ein, in der Hoffnung, dadurch die Diskussion abzukürzen, indem ich annehmen will, daß er jene, für seine Vorschläge ja gar nicht erheblichen, irrigen Darstellungen meiner Ansichten stillschweigend fallen lassen wird.

Zürich, 16./10. 1906 .

\section{Sitzungsberichte.}

Fïnfte Versammlung der internationalen Kommission für einheitliche Methoden der Zuckeruntersuchung in Bern 1906 .

Am 3. und 4./8.1906 fand in Bern die fünfte Versammlung der genannten Kommission statt. Den Vorsitz führte Prof. A. H e r z f e ld - Berlin als Vertreter des Vereins der deutschen Zuckerindustrie. Weiter waren vertreten Osterreich-Ungarn durch Regierungsrat F. S t roh me r - Wien, Frankreich durch Saillard, Belgien duroh F. Sachs, RuBland durch Prof. Schukow, die javanische Zuckerindustrie durch P rins e n. $\mathrm{G}$ e e r lig s, die engliche durch $W$ a $\mathrm{t} t$ - Liverpool und $\mathrm{Ma}$ in - London und die amerikanische durch Wi e c h m an n - Neu-York .

Den ersten Gegenstand der Tagesordnung bildete die Festsetzung einer einheitlichen Bereitungsvorschrift für die Fehling sche Lösung, sowie der Art der Invertzuckerbestimmung. Das Referat darüber führten $\mathrm{W}$ a $\mathrm{t} t$, W i e chm an $\mathrm{n}$ und.
Strohmer. Es handelte sich hier um die Klä rung der Lösung vor Ausführung der Invertzuckerbestimmung, welche von den englischen Chemikern nicht vorgenommen wird und als Ursache der Differenz zwischen den englischen und kontinentalen Analysenergebnissen gilt. Zur Lösung dieser Frage wird eine Kommission eingesetzt, welcher angehören Herzfold, Strohmer, Saillard, Wat $t$, Main, Schukow und v. Buchka.

W i o o h ma $n \mathbf{n}$ gibt den Bericht über, ,einheitliche internationale Vorschriften für die Probe. nahme der Zuckerprodukte“. Stroh mer will die Frage der Musterziehung nicht der Kommission, sondern den Vertretern des Handels zur Lösung vorgelegt wissen. S a i 11 a r d berichtet uiber eine einheitliche Form und Ausdruckswejse der Analysenzertifikate für den internationalen Zucker. handel. Zu den beiden Referaten wird ein einheitlicher Beschlu $B$ nicht gefaßt. W i e e h m a $n \mathrm{n}$ berichtet über die Vermeidung des Niederschlags- 